\title{
Substantial pressure effect on the resistivity and Curie temperature for the diluted magnetic semiconductor $\mathrm{Sb}_{2-x} \mathrm{~V}_{x} \mathrm{Te}_{3}$
}

\author{
J. S. Dyck ${ }^{*, 1}$, K. Ahilan ${ }^{* *, 2}$, M. C. Aronson ${ }^{2}$, C. Uher $^{2}$, and P. Lošt’ák ${ }^{3}$ \\ 1 Department of Physics, John Carroll University, University Heights, OH 44118 \\ ${ }^{2}$ Department of Physics, University of Michigan, Ann Arbor, MI 48109, USA \\ ${ }^{3}$ Faculty of Chemical Technology, University of Pardubice, Čs. Legií Square 565, 53210 Pardubice, \\ Czech Republic
}

Received 13 September 2005, revised 7 February 2006, accepted 10 March 2006 Published online 5 May 2006

PACS 72.80.Jc, 75.30 Et, 75.30.Kz, 75.50.Pp, 85.75.-d

The influence of hydrostatic pressure on the electrical resistivity $\rho$ and ferromagnetic transition temperature $T_{\mathrm{C}}$ of bulk, single crystal $\mathrm{Sb}_{1-r} \mathrm{~V}_{r} \mathrm{Te}_{3}$ with $x=0.03$ is presented. Pressure strongly suppresses $\rho$ at all temperatures, with an overall decrease of about $35 \%$ at $1.6 \mathrm{GPa}$. The peak in $\rho$, a signature of $T_{\mathrm{C}}$, moves to lower temperature with increasing pressure. An overall suppression of $T_{\mathrm{C}}$ near $40 \%$ at $1.6 \mathrm{GPa}$ is observed. The results are discussed within the context of a carrier-mediated ferromagnetic exchange interaction.

(C) 2006 WILEY-VCH Verlag GmbH \& Co. KGaA, Weinheim

\section{Introduction}

Diluted magnetic semiconductors (DMS) continue to be of interest to the scientific community both for their fundamental scientific interest and for potential applications [1]. Although no theoretical consensus on the precise ferromagnetic mechanism has yet been reached in the literature, it is accepted that free charge carriers in the semiconductor host mediate the interaction between magnetic ions [2-4], at least in III-V based DMS doped with Mn [5-8]. Techniques commonly used to characterize diluted magnetic semiconductors include electrical transport and magnetization carried out at atmospheric pressure. For a broader understanding of ferromagnetism in DMS, alternative DMS structures and different characterization techniques should be investigated.

Vanadium-doped $\mathrm{Sb}_{2} \mathrm{Te}_{3}$ is one such alternative ferromagnetic DMS that can be prepared in single crystal form [9]. The origin of the magnetic order in this material has so far not been established. Antimony telluride is a narrow band gap semiconductor $\left(E_{\mathrm{g}}=0.26 \mathrm{eV}\right)$ and possesses the layered, tetradymite structure. A background free hole concentration of $1 \times 10^{20} \mathrm{~cm}^{-3}$ arising from native antisite defects is typical for pure $\mathrm{Sb}_{2} \mathrm{Te}_{3}$, and doping with vanadium does not alter the carrier concentration. The valence band of the host semiconductor is multi-valley $[10,11]$ and there is evidence from Shubnikov-de Haas data that both an upper, light hole and lower, heavy hole band are populated for these high hole densities [12]. The pressure dependence of electrical resistivity in $\mathrm{Sb}_{2} \mathrm{Te}_{3}$ [13] and isostructural $\mathrm{Bi}_{2} \mathrm{Te}_{3}$ [14] has been investigated previously, and resistivity was found to be significantly reduced upon application of

\footnotetext{
* Corresponding author: e-mail: jdyck@jcu.edu, Phone: +00 1216397 4560, Fax: +00 12163974499

** Present address: Department of Physics, University of Jaffna, Sri Lanka 
high pressures. This fact suggests that high pressure studies may be a way to probe the nature of a potential carrier-mediated ferromagnetic order in $\mathrm{Sb}_{2-x} \mathrm{~V}_{x} \mathrm{Te}_{3}$.

The magnetic phenomena of DMS structures that exhibit paramagnetic or spin glass phenomena have been studied with the aid of high pressures [15], but there are very few reports in the literature on the pressure dependence of the ferromagnetic transition in a DMS material [16]. In bulk single crystals of $(\mathrm{Pb}, \mathrm{Sn})_{1-x} \mathrm{Mn}_{x} \mathrm{Te}$, a IV - VI DMS where the ferromagnetic interaction is governed by the carrier-mediated RKKY interaction [17], high-pressure measurements helped to elucidate the relationship between $T_{\mathrm{C}}$ and the underlying band structure [16]. Recently, it was observed that application of high pressures increases $T_{\mathrm{C}}$ in $\mathrm{In}_{1-x} \mathrm{Mn}_{x} \mathrm{Sb}$ thin films [18].

In this report, we present the results of electrical resistivity measurements on bulk, single crystal $\mathrm{Sb}_{2-x} \mathrm{~V}_{x} \mathrm{Te}_{3}$ with $x=0.03$ for applied hydrostatic pressures of up to $1.6 \mathrm{GPa}$. We find that modest pressures have a large effect on both the resistivity $\rho$ and the Curie temperature $T_{\mathrm{C}}$ in this material which suggests that a carrier mediated interaction is responsible for the ferromagnetic order.

\section{Experimental}

Single crystal $\mathrm{Sb}_{2-x} \mathrm{~V}_{x} \mathrm{Te}_{3}$ with $x$ near 0.03 was prepared using the Bridgman method. The composition and phase purity were verified with electron microprobe analysis and X-ray crystallography. Resistance versus temperature data, with current perpendicular to the trigonal $c$-axis, was taken in a standard fourprobe configuration with a lock-in amplifier operating at $48 \mathrm{~Hz}$. The specimen was loaded into a liquid clamp pressure cell (pressure transmitting fluid was $3 \mathrm{M}^{\mathrm{TM}}$ Fluorinert ${ }^{\mathrm{TM}}$ Electronic Liquid FC-77), and pressures were determined in situ using a superconducting lead manometer [19].

\section{Results}

We have established previously [9] that $T_{\mathrm{C}}$ in $\mathrm{Sb}_{2-x} \mathrm{~V}_{x} \mathrm{Te}_{3}$ increases with $x$ and reaches $\sim 22 \mathrm{~K}$ for $x=0.03$ (highest attainable value of $x$ in bulk crystals). Figure 1 displays the temperature dependence of both the electrical resistivity $\rho$ (open symbols) and magnetization $M$ (solid symbols) at ambient pressure for a representative sample with $x$ near 0.03 . A peak in the resistivity $\rho_{\text {peak }}$ develops near the temperature where the point of inflection of the $M$ versus $T$ curve occurs. This correlation is observed for all samples regardless of vanadium content, and therefore $\rho_{\text {peak }}$ can serve as a signature of the onset of the ferromagnetic state. We define the Curie temperature $T_{\mathrm{C}}$ to be this point.

Electrical resistivity data taken at different pressures for single crystal $\mathrm{Sb}_{1.97} \mathrm{~V}_{0.03} \mathrm{Te}_{3}$ are presented in Fig. 2. Data from crystals with somewhat lower vanadium concentrations are qualitatively the same. At ambient pressure, $\rho$ has the metallic temperature dependence characteristic of a degenerate p-type semi-

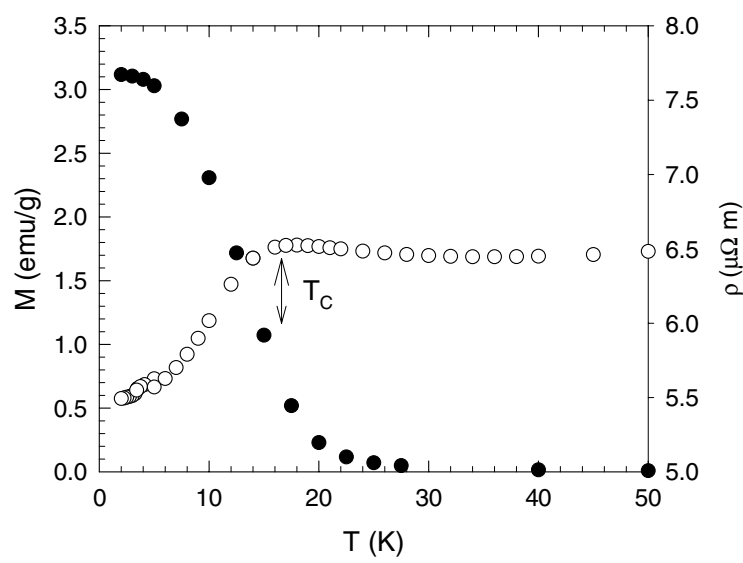

Fig. 1 Electrical resistivity $\rho$ (open symbols) and magnetism $M$ (solid symbols) of a single crystal of $\mathrm{Sb}_{1.97} \mathrm{~V}_{0.03} \mathrm{Te}_{3}$ as a function of temperature near the Curie temperature $T_{\mathrm{C}}$. The peak in resistivity occurs very near $T_{\mathrm{C}}$. 


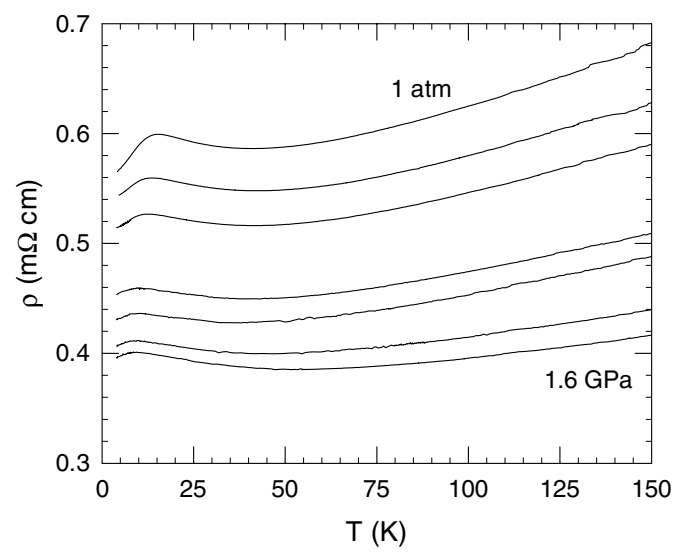

Fig. 2 Electrical resistivity $\rho$ versus temperature for single crystal $\mathrm{Sb}_{1.97} \mathrm{~V}_{0.03} \mathrm{Te}_{3}$ taken at applied pressures of up to 1.6 GPa. Successive curves represent pressures of 1 atm (negligible applied pressure), $0.15 \mathrm{GPa}, 0.20 \mathrm{GPa}, 0.76 \mathrm{GPa}$, 1.0 GPa, 1.4 GPa and 1.6 GPa.

conductor. One of the major effects of increasing pressure is the strong reduction of resistivity at all temperatures. At a pressure of $1.6 \mathrm{GPa}$, the reduction in $\rho$ near $T_{\mathrm{C}}$ is $\sim 35 \%$ for our vanadium-doped sample, which is very similar to the magnitude of the reduction in pure $\mathrm{Sb}_{2} \mathrm{Te}_{3}$ [13]. Given this similar effect, we suppose that it is primarily a characteristic of the host band structure that is responsible for the decrease in resistivity. If we adopt the model that holes from both an upper (high mobility) and lower (low mobility) valence band participate in the conduction [12], the pressure dependence of the band structure would be important when considering this reduction effect. In isostructural $\mathrm{Bi}_{2} \mathrm{Te}_{3}$, a similar reduction in $\rho$ with pressure was interpreted by assuming the total number of free holes was constant and, upon application of pressure, heavy holes from the lower valence band filled the upper hole band as the energy separation between the valence band extrema increased [14].

A second feature in the data is that the peak in the resistivity $\rho_{\text {peak }}$, our signature of $T_{\mathrm{C}}$, moves to lower temperature with increasing pressure. Figure 3(a) shows $\rho / \rho_{\text {peak }}$ versus $T$ at low temperatures for several pressures, and the arrows indicate the location of $T_{\mathrm{C}}$. The dependence of $T_{\mathrm{C}}$ and $\rho_{\text {peak }}$ on pressure is displayed in Fig. 3(b). For small pressures, $\mathrm{d} T_{\mathrm{C}} / \mathrm{d} P=-13 \mathrm{~K} / \mathrm{GPa}$, and then the magnitude of $\mathrm{d} T_{\mathrm{C}} / \mathrm{d} P \mathrm{de}-$ creases for higher pressures. At a pressure of $1.0 \mathrm{GPa}$, the reduction amounts to $35 \%$ relative to the ambient pressure value. We note that the influence of pressure on $T_{\mathrm{C}}$ and $\rho_{\text {peak }}$ is quite similar.
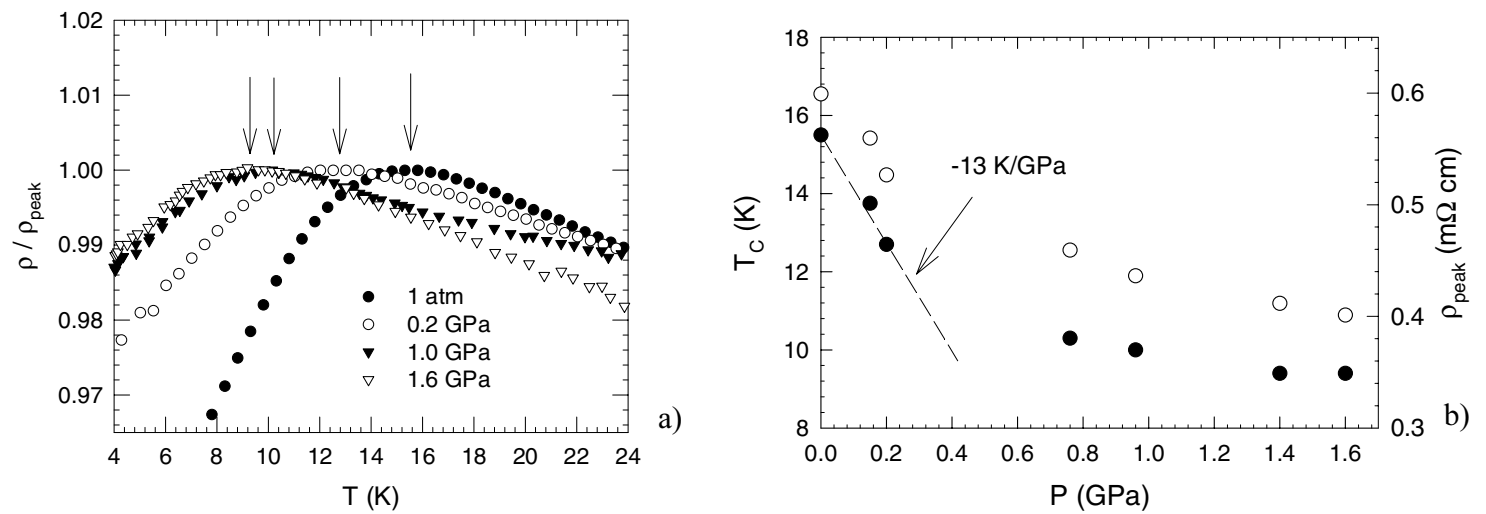

Fig. 3 a) Resistivity $\rho$ normalized to its value at $\rho_{\text {peak }}$ (indicated by the arrows) versus temperature for several pressures. b) Pressure dependence of $\rho_{\text {peak }}$ (open symbols) and the Curie temperature $T_{\mathrm{C}}$ (solid symbols). 


\section{Discussion}

In order to understand the large suppression of $T_{\mathrm{C}}$ upon applied pressure, we must consider which parameters are most likely affected by a change in volume. A Zener-RKKY mean field model has been used to describe the (III,Mn) V systems in the strongly degenerate limit provided the concentration of impurities is much greater than the concentration of holes [2]. Within this model, for a single parabolic valence band, the Curie temperature can be expressed as

$$
T_{\mathrm{C}} \propto N S(S+1) J_{\mathrm{pd}}^{2} m_{\mathrm{eff}} k_{\mathrm{F}},
$$

where $N$ is the concentration of magnetic ions, $S$ is the ion spin, $J_{\mathrm{pd}}$ is the local p-d exchange interaction, $m_{\text {eff }}$ is the hole effective mass, and $k_{\mathrm{F}}$ is the Fermi wave vector. Antiferromagnetic superexchange interactions between magnetic ions are ignored here. In a two valence band model, Eq. (1) would apply separately to light holes and to heavy holes. We consider the possibility that this model might be applicable in $\mathrm{Sb}_{2-x} \mathrm{~V}_{x} \mathrm{Te}_{3}$. The atmospheric pressure hole concentration as determined by $1 /\left(R_{\mathrm{H}} e\right)$, where $R_{\mathrm{H}}$ is the Hall coefficient in low field and $e$ is the electronic charge, is $1 \times 10^{20} \mathrm{~cm}^{-3}$, and we take this value as an estimate of the total number of carriers in both valence bands as has been done in Ref. [20]. Taking into account the six-fold degeneracy of the valence bands, we expect a hole concentration per carrier pocket of roughly $2 \times 10^{19} \mathrm{~cm}^{-3}$. For a vanadium content corresponding to $x=0.03, N \approx 2 \times 10^{20} \mathrm{~cm}^{-3}$. Therefore, we argue that the ferromagnetic order with $T_{\mathrm{C}}$ described by Eq. (1) could be appropriate for $\mathrm{Sb}_{2-x} \mathrm{~V}_{x} \mathrm{Te}_{3}$, at least at atmospheric pressure.

Using available data [21] for the hydrostatic pressure dependence of the lattice parameters for $\mathrm{Sb}_{2} \mathrm{Te}_{3}$, the reduction in volume is roughly $4 \%$ at the highest pressures, which would increase $N$ by $\sim 4 \%$ leading to an increase in $T_{\mathrm{C}}$. In $\mathrm{Sb}_{1.97} \mathrm{~V}_{0.03} \mathrm{Te}_{3}$, vanadium takes the $\mathrm{V}^{3+}$ state with $S=1$ [9]. A reduction in the spin-value would imply a $V^{4+}$ state, which is unlikely given that this impurity would be a donor. These donor states would compensate the holes leading to an increase in resistivity, rather than the decrease that is observed. In principle, the exchange interaction $J_{\text {pd }}$ is expected to be somewhat enhanced with pressure; indeed, in the (III,Mn)-V based DMS compounds, $J_{\mathrm{pd}} \sim V^{-1}[2,18]$. The net effect of increasing the pressure for each of the parameters discussed above should lead to a substantial increase in $T_{\mathrm{C}}$, contrary to the observed trend.

The decrease in resistivity, and its relation to hole concentration, is likely key to understanding this anomalous decrease in $T_{\mathrm{C}}$. It is possible that Eq. (1) may become invalid if carrier concentration increases with increased pressure. For high enough hole densities, RKKY oscillations would lead to frustrated indirect coupling between $V$ local moments and to a suppression of $T_{\mathrm{C}}$. The pressure dependence of native defect or impurity states in $\mathrm{Sb}_{2} \mathrm{Te}_{3}$ is unknown, so it is not clear how the overall hole concentration could increase. An alternative explanation is that there is a redistribution of heavy holes and light holes among the valence bands as was done in p-type $\mathrm{Bi}_{2} \mathrm{Te}_{3}$ [14]. Such a picture was invoked to explain the pressure dependence of $T_{\mathrm{C}}$ in $(\mathrm{Pb}, \mathrm{Sn})_{1-x} \mathrm{Mn}_{x} \mathrm{Te}$ where the heavy holes alone mediated the ferromagnetic interaction [17]. Without further study, it is not possible to discern which model is more likely. Detailed studies of magnetoresistance and Hall effect under pressure would further elucidate the behavior of the band parameters.

Regardless of the exact mechanism of magnetic interaction, the data point to the fact that a small change in the lattice parameter in $\mathrm{Sb}_{1.97} \mathrm{~V}_{0.03} \mathrm{Te}_{3}$ has a dramatic effect on the Curie temperature. Based on the results, we postulate that an increase in lattice parameter for vanadium-doped tetradymite structure semiconductor hosts could lead to higher $T_{\mathrm{C}}$. Strain induced through epitaxial growth techniques provides a means to explore this avenue. Moreover, high pressure experiments can be a powerful way to explore the relationship between electrical and magnetic properties in these and other ferromagnetic DMS materials.

\section{Summary}

In summary, we find an unusually large pressure effect on the electrical resistivity and Curie temperature in vanadium doped $\mathrm{Sb}_{2} \mathrm{Te}_{3}$. Both $\rho$ and $T_{\mathrm{C}}$ decrease with increasing pressure and $\left|\mathrm{d} T_{\mathrm{C}} / \mathrm{d} P\right|$ decreases with 
increasing pressure. The large reduction in resistivity suggests a large change in carrier concentration, valence band structure, or both. Further study on the pressure dependence of the carrier concentration will likely shed more light on the fundamental nature of the supposed carrier-mediated ferromagnetic state. This work establishes pressure as an important experimental variable for study in ferromagnetic DMS materials.

Acknowledgements We gratefully acknowledge the support provided by the National Science Foundation under grant INT 0201114 (JSD and CU) and DMR 0305221 (CU), the Ministry of Education of Czech Republic under the project MSM 0021627501 (PL), the Research Corporation through award CC6035 (JSD), and the U. S. Department of Energy, Office of Basic Energy Sciences under grant DE-FG02-94ER45526 (MCA and KA).

\section{References}

[1] G. A. Prinz, Science 282, 1660 (1998).

[2] T. Dietl, H. Ohno, F. Matsukura, J. Cibert, and D. Ferrand, Science 287, 1019 (2000).

[3] T. Jungwirth, J. Konig, J. Sinova, J. Kucera, and A. H. MacDonald, Phys. Rev. B 66, 012402 (2002).

[4] S. Das Sarma, E. H. Hwang, and D. J. Priour, Jr., Phys. Rev. B 70, 161203(R) (2004).

[5] H. Ohno, A. Shen, F. Matsukura, A. Oiwa, A. Endo, S. Katsumoto, and Y. Iye, Appl. Phys. Lett. 69, 363 (1996).

[6] S. J. Potashnik, K. C. Ku, S. H. Chun, J. J. Berry, N. Samarth, and P. Schiffer, Appl. Phys. Lett. 79, 1495 (2001).

[7] K. C. Ku, S. J. Potashnik, R. F. Wang, S. H. Chun, P. Schiffer, N. Samarth, M. J. Seong, A. Mascarenhas, E. Johnston-Halperin, R. C. Myers, A. C. Gossard, and D. D. Awschalom, Appl. Phys. Lett. 82, 2302 (2003).

[8] Sh. U. Yuldashev, H. Im, V. Sh. Yalishev, C. S. Park, T. W. Kang, S. Lee, Y. Sasaki, X. Liu, and J. K. Furdyna, Appl. Phys. Lett. 82, 1206 (2003).

[9] J. S. Dyck, P. Hajek, P. Lostak, and C. Uher, Phys. Rev. B 65, 115212 (2002).

[10] A. Von Mittendorf, K. Dietrich, and G. Lanwehr, Solid State Commun. 3, 443 (1973).

[11] P. Larson, Phys. Rev. B 68, 155121 (2003).

[12] V. A. Kulbachinskii, Z. M. Dashevskii, M. Inoue, M. Sasaki, H. Negishi, W. X. Gao, P. Lostak, J. Horak, and A. de Visser, Phys. Rev. B 52, 10915 (1995).

[13] L. G. Khvostantsev, A. I. Orlov, N. Kh. Abrikosov, and L. D. Ivanova, phys. stat. sol. (a) 58, 37 (1980).

[14] N. B. Brandt and V. A. Kulbachinkii, Semicond. Sci. Technol. 7, 907 (1992).

[15] N. Kuroda, Semiconductors and Semimetals Vol. 54 (Academic Press, New York, 1998), pp. 513-550, and references therein.

[16] T. Story, R. R. Galazka, R. B. Frankel, and P. A. Wolff, Phys. Rev. Lett. 56, 777 (1986).

[17] T. Suski, J. Igalson, and T. Story, J. Magn. Magn. Mater. 66, 325 (1987).

[18] M. Csontos, G. Mihaly, B. Janko, T. Wojtowicz, X. Liu, and J. K. Furdyna, Nature Mater. 4, 447 (2005).

[19] T. F. Smith, C. W. Chu, and M. B. Maple, Cryogenics 9, 53 (1969).

[20] V. A. Kulbachinskii, N. Miura, H. Nakagawa, C. Drasar, and P. Lostak, J. Phys.: Condens. Matter 11, 5273 (1999).

[21] N. Sakai, T. Kajiwara, K. Takemura, S. Minomura, and Y. Fujii, Solid State Commun. 40, 1045 (1981). 